\title{
Acute Childhood Cardiorenal Syndrome and Impact of Cardiovascular Morbidity on Survival
}

\author{
Wasiu A. Olowu \\ Paediatric Nephrology and Hypertension Unit, Obafemi Awolowo University Teaching Hospitals Complex, PMB 5538, Ile-Ife, \\ Osun State 234, Nigeria \\ Correspondence should be addressed to Wasiu A. Olowu, yetundeolowu@yahoo.com
}

Received 15 August 2010; Accepted 21 March 2011

Academic Editor: Anjay Rastogi

Copyright ( $(2011$ Wasiu A. Olowu. This is an open access article distributed under the Creative Commons Attribution License, which permits unrestricted use, distribution, and reproduction in any medium, provided the original work is properly cited.

Cardiorenal syndrome (CRS) clinical types, prevalence, aetiology, and acute cardiovascular morbidity impact on the outcome of acute kidney function perturbation were determined. Forty-seven of 101 (46.53\%) patients with perturbed kidney function had CRS. Types 3 and 5 CRS were found in 10 and 37 patients, respectively. Type 3 CRS was due to acute glomerulonephritis (AGN; $n=7)$, captopril $(n=1)$, frusemide $(n=1)$, and hypovolaemia $(n=1)$. Malaria-associated haemoglobinuria $(n=20)$, septicaemia $(n=11)$, lupus nephritis $(n=3)$, tumour lysis syndrome $(n=2)$, and acute lymphoblastic leukaemia $(n=1)$ caused Type 5 CRS. The cumulative mortality in hypertensive CRS was similar to nonhypertensive CRS (51.4\% versus $40.9 \% ; P=.119)$. Mortality in CRS and non-CRS was similar $(45.7 \%$ versus $24.5 \% ; P=.053)$. Type 5 survived better than type 3 CRS $(66.7 \%$ versus $12.5 \% ; P=.001)$. Risk factors for mortality were Type 3 CRS $(P=.001)$, AGN-associated CRS $(P=.023)$, dialysis requiring CRS $(P=.008)$, and heart failure due to causes other than anaemia $(P=.003)$. All-cause-mortality was $34.2 \%$. Preventive measures aimed at the preventable CRS aetiologies might be critical to reducing its prevalence.

\section{Introduction}

The cardiorenal syndrome (CRS) is a disorder of the heart and kidneys whereby acute or chronic dysfunction in one organ may induce acute or chronic dysfunction of the other $[1,2]$; it is a recognized morbidity and mortality multiplier in critically ill children [3]. While heart failure (HF) is a clinical syndrome in which heart disease reduces cardiac output, increases venous pressures, and is accompanied by molecular abnormalities that cause progressive deterioration of the failing heart and premature myocardial cell death [4], acute kidney injury (AKI) is an abrupt clinical and/or laboratory manifestation of kidney dysfunction usually within 48 hours of bilateral kidney insult of any kind. Failure of both organs commonly coexists in critically ill children [5-7]. Congestive HF is a highly prevalent AKI comorbidity and a major indication for acute dialysis in children [5]. Recently, the 7th Acute Dialysis Quality Initiative (ADQI) workgroup classified CRS into five distinct clinical types, $[1,2]$ namely: acute CRS (Type 1) -acute worsening of heart function leading to kidney injury and/or dysfunction; chronic CRS
(Type 2)—chronic abnormalities in heart function leading to kidney injury and/or dysfunction; acute renocardiac syndrome (Type 3)-acute worsening of kidney function (AKI) leading to heart injury and/or dysfunction; chronic renocardiac syndrome (Type 4) — chronic kidney disease leading to heart injury, disease, and/or dysfunction, and secondary CRS (Type 5) - systemic conditions leading to simultaneous injury and/or dysfunction of heart and kidney. While a lot of data have been published on chronic kidney disease as risk factor for cardiovascular morbidity and mortality in both children and adults (reviewed in [1-3]), there is paucity of specific data on acute cardiac dysfunction leading to AKI and vice versa in children especially [8]; in this study, an attempt was made to determine the prevalence, aetiology, clinical types of CRS, and impact of acute cardiovascular morbidity on the outcome of childhood acute kidney injury.

\section{Patients and Methods}

Clinical charts of patients managed for AKI-associated HF and acute glomerulonephritis (AGN) - associated HF in our 
paediatric nephrology and hypertension unit were reviewed. It was a retrospective case-control study; patients who had either AKI or AGN without HF served as control (non-CRS). The objectives were to determine the prevalence, aetiology, clinical types of CRS, and impact of acute cardiovascular morbidity on the outcome of childhood AKI. The study period ranged between January 2005 and December 2009. Our hospital's Ethics and Research Committee approved the research protocol. The study conformed to the provisions of the revised Declaration of Helsinki, Edinburg, 2000.

Analyzed data were age, gender, anthropometry, vital signs, admission diagnosis, time of onset of HF and AKI, final AKI stage, hospitalization period, follow-up duration, urine output, and management outcome. Relevant laboratory investigations including serum creatinine (Scr) both at baseline and at followup were reviewed.

2.1. Definitions. AKI was diagnosed based on the acute kidney injury network (AKIN) criteria [9] as an absolute increase in serum creatinine (Scr) level within 48 hours of bilateral kidney insult by $\geq 0.3 \mathrm{mg} / \mathrm{dL} \quad(\geq 26.4 \mu \mathrm{mol} / \mathrm{L})$ or a $50 \%(1.5$-fold) increase or more in Scr from the baseline. AKI was staged using the creatinine criteria of the AKIN workgroup [9]—Stage 1 AKI (AKI-1): rise in Scr by $\geq 0.3 \mathrm{mg} / \mathrm{dL}(26.4 \mu \mathrm{mol} / \mathrm{L})$ or an increase of $>150$ $200 \%$ (1.5- to 2-fold increase) from the baseline; Stage 2 AKI (AKI-2): rise in Scr by $>200-300 \%$ ( $>2$ - to 3 -fold increase) from baseline; Stage 3 AKI (AKI-3): rise in Scr by $>300 \%$ ( $>3$-fold) from the baseline or Scr $\geq 4.0 \mathrm{mg} / \mathrm{dL}$ $(\geq 354 \mu \mathrm{mol} / \mathrm{L})$ with an acute rise of at least $0.5 \mathrm{mg} / \mathrm{dL}$ $(44 \mu \mathrm{mol} / \mathrm{L})$. Nonoliguric AKI was defined as urine output that was persistently $>0.5 \mathrm{~mL} / \mathrm{kg} / \mathrm{hour}$ in the setting of an abnormal Scr level. Anuric AKI was defined as the urine output that was $<0.039 \mathrm{~mL} / \mathrm{kg} / \mathrm{hr}$ for $12 \mathrm{hr}$ or more in the absence of an obstructive uropathy. AKI was staged based on the peak Scr (pScr) level. pScr was the highest Scr level reached in any patient either before death or before gradual return to normal. Those who were initially diagnosed AKI1 or AKI-2 but later required dialyses were upgraded to AKI-3 as recommended [9]. The predictive eGFR equation with corrections for age, gender, and race derived by the Modification of Diet in Renal Disease (MDRD) [10] study group was used to determine the baseline Scr for patients who do not have baseline Scr as recommended by the 2nd ADQI workgroup [11]. For such patients, the 2nd ADQI recommended that normal estimated glomerular filtration rate (eGFR) ranging from 75 to $100 \mathrm{~mL} / \mathrm{min}$ per $1.73 \mathrm{~m}^{2}$ should be used. In this study, all AKI patients without baseline measure of renal function were assumed to have eGFR value of $100 \mathrm{~mL} / \mathrm{min} / 1.73 \mathrm{~m}^{2}$. By the MDRD equation, $\mathrm{eGFR}=186 \times\left([\mathrm{Scr}]^{-1.154} \times \mathrm{Age}^{-0.203} \times 0.742(\right.$ if female $) \times$ 1.210 (if black)) [10].

Heart failure was diagnosed based on a combination of dyspnoea, tachycardia (heart rate $>160,>150,>140,>120$, and $>100$ beats/min for infants, children aged $1-3,4-5,6-12$, and above 12 years, resp.), tachypnoea (respiratory rate $>60$, $>40,>34,>30$, and $>20$ breathes/min for infants, children aged $1-3,4-5,6-12$, and above 12 years, resp.), tender hepatomegaly, and feeding difficulty with or without chest
$\mathrm{X}$-ray evidence of cardiomegaly (abnormal cardiothoracic ratio $>60 \%$ in under fives and $>55 \%$ in older children). HF severity was assessed and classified according to the modified Ross heart failure classification for children [12] Class I heart failure: asymptomatic; Class II heart failure: mild tachypnoea or diaphoresis with feeding in infants or dyspnoea on exertion in older children; Class III heart failure: marked tachypnoea or diaphoresis with feeding in infants, marked dyspnoea on exertion, and prolonged feeding times with growth failure; Class IV heart failure: symptoms such as tachypnoea, retractions, grunting, or diaphoresis at rest. Hypertension was diagnosed based on the update of the 1987 Task Force Report on High Blood Pressure in Children and Adolescents [13]. CRS was classified based on the 7th ADQI consensus conference report [2].

The inclusion criteria were patients with acute perturbation of kidney function (AKI or AGN or both) with or without HF. Patients with chronic renal failure or acute-onchronic renal failure were excluded. To determine the impact of HF on survival, mortality was compared between CRS $(\mathrm{AKI}+\mathrm{HF}$ and AGN + HF) and non-CRS patients. The cumulative all-cause-mortality and CRS-specific mortality rates were determined.

2.2. Statistical Analysis. Descriptive statistics used comprised mean, standard deviation, median, percentages, and proportions. The comparative statistics were Student's $t$-test, Chi-square test, Cox regression analysis for hazard ratio (HR), Wilcoxon statistics, Kaplan-Meier survival analysis, and Mantel-Cox pairwise comparisons (Log-rank test) using the SPSS 15.0 for Windows evaluation version (2006, SPSS Inc.). A $P$-value $<.05$ was regarded as statistically significant.

\section{Results}

A total of 101 patients with acute perturbation of kidney function, namely, AKI and AGN were reviewed. There were 7 and 94 acute glomerulonephritis (AGN) and AKI patients, respectively. Forty seven of $101(46.53 \%)$ patients with abnormal kidney function had HF-cardiorenal syndrome. HF was of the severest class (Class IV) in all the CRS patients. Age, gender, and blood pressure data are summarized in Table 1. Median age of 5.0 years (0.06-15.0) for controls was similar to that for CRS, $P=.689$. Types 3 and 5 CRS were found in $10(21.3 \%)$ and $37(78.7 \%)$ patients, respectively. Table 2 shows the relationship between the two CRS types in this study and their aetiologies. Two of 7 patients whose CRS was due to AGN had no associated AKI (AGN-AKI) while the remaining 5 had associated AKI. The pScr was $6.11 \pm 4.0(0.95-17.32) \mathrm{mg} / \mathrm{dL}$. AKI-1, AKI-2, and AKI-3 accounted for $4(8.50 \%), 5(10.60 \%)$, and $36(76.60 \%)$ CRS cases, respectively, while AGN-AKI accounted for the rest. Twenty-two (46.80\%) of the CRS patients had oliguric AKI while nonoliguric and anuric AKI were seen in $11(23.40 \%)$ and $14(29.80 \%)$ patients, respectively. Oliguria duration in both CRS and controls was $6.9 \pm 5.54$ days and $7.5 \pm 4.75$ days, respectively $(P=.654)$.

Anaemia was present in 43 of 47 CRS patients (91.5\%). The haematocrit ranged from 4.0 to $32.0 \%$ with the 5 th, 
TABLE 1: Demographic and clinical characteristics of the cardiorenal syndrome patients $(n=47)$.

\begin{tabular}{|c|c|}
\hline $\begin{array}{l}\text { Demographic and baseline clinical } \\
\text { characteristics }\end{array}$ & Results (\%) \\
\hline Age $<6$ years & $33(70.21)$ \\
\hline Age $\geq 6$ years & $14(29.79)$ \\
\hline Median age (range), years & $4.0(.3-14.5)$ \\
\hline \multicolumn{2}{|l|}{ Gender } \\
\hline Male & $26(55.32)$ \\
\hline Female & $21(44.68)$ \\
\hline Male to female ratio & $1.24: 1$ \\
\hline Number with normal blood pressure $(\mathrm{BP})^{\mathrm{a}}$ & $26(57.8)$ \\
\hline Systolic BP range, $\mathrm{mmHg}$ & $60-110$ \\
\hline 5 th, 50th, and 95 th percentiles in $\mathrm{mmHg}$ & $63.5,90$, and 110 \\
\hline Diastolic $\mathrm{BP}$ range, $\mathrm{mmHg}$ & $30-70$ \\
\hline 5 th, 50th, and 95 th percentiles in $\mathrm{mmHg}$ & $33.5,50$, and 70 \\
\hline Mean arterial pressure range, $\mathrm{mmHg}$ & $43.3-83.3$ \\
\hline \multicolumn{2}{|c|}{ 5th, 50th, and 95th percentiles in $\mathrm{mmHg} 44.49,66.7$, and 82.8} \\
\hline Number with hypertension & $19(42.2)$ \\
\hline Systolic BP range, $\mathrm{mmHg}$ & $90-190$ \\
\hline 5 th, 50th, and 95 th percentiles in $\mathrm{mmHg}$ & 90,120 , and 190 \\
\hline Diastolic BP range, $\mathrm{mmHg}$ & $60-130$ \\
\hline 5 th, 50th, and 95th percentiles in $\mathrm{mmHg}$ & 60,80 , and 130 \\
\hline Mean arterial pressure range, $\mathrm{mmHg}$ & $73.3-150$ \\
\hline 5th, 50th, and 95th percentiles in $\mathrm{mmHg}$ & $73.3,93.3$, and 150 \\
\hline
\end{tabular}

TABLE 2: Relation between cardiorenal syndrome types and their aetiologies.

\begin{tabular}{lc}
\hline Cardiorenal syndrome type and aetiology & $\begin{array}{c}\text { Proportion } \\
\text { of patients } \\
(\%)\end{array}$ \\
\hline $\begin{array}{l}\text { Type 3 } \\
\text { Acute glomerulonephritis }\end{array}$ & $7.0(70.0)$ \\
Captopril & $1.0(10.0)$ \\
Frusemide & $1.0(10.0)$ \\
Hypovolaemic shock due to gastroenteritis & $1.0(10.0)$ \\
\hline Type 5 & $20.0(54.05)$ \\
Malaria-associated haemoglobinuria & $11.0(29.73)$ \\
Septicaemia & $3.0(8.11)$ \\
Lupus nephritis & $2.0(5.41)$ \\
Tumour lysis syndrome in Burkitt's lymphoma & $1.0(2.70)$ \\
patients & Acute lymphoblastic leukaemia
\end{tabular}

50th, and the 95th haematocrit percentiles for the anemic CRS being $5.2 \%, 15.0 \%$, and $27.6 \%$, respectively. Severe, moderate, and mild anaemia occurred in $38(88.37 \%), 2$ $(4.65 \%)$, and $3(6.98 \%)$ patients, respectively, $(P=.000)$. Malaria-associated haemoglobinuria (MAH), septicaemia, AGN, lupus nephritis and tumour lysis syndrome accounted

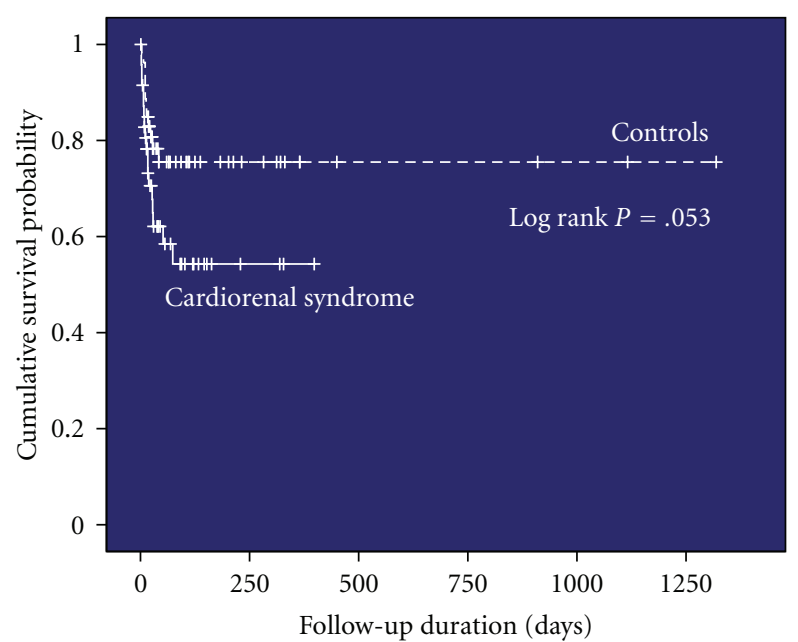

FIGURE 1: Kaplan-Meier survival curves showing that survival was not significantly higher in controls than in cardiorenal syndrome $(75.5 \%$ versus $54.3 \%)$. Similarly, the survival times were not significantly different.

for $20(46.51 \%), 10(23.25 \%), 4(9.30 \%), 3(6.97 \%)$, and $2(4.65 \%)$ anaemia cases, respectively. Hypovolaemia due to gastroenteritis, acute lymphoblastic leukaemia, captopril, and frusemide accounted for one $(2.33 \%)$ case each, $(P=$ $.000)$. Anaemia was absent in 1 septicaemia and 3 AGN cases.

3.1. Followup and Outcome. The mean CRS hospitalization period was $21.90 \pm 16.42$ days (controls: $25.1 \pm 19.7 ; P=$ $.361)$. Both the CRS and non-CRS patients were, respectively, followed for $67.2 \pm 90.97(1.0-398.0)$ days and $128.96 \pm$ $240.61(1.0-1319.0 ; P=.106)$ days. Survival in CRS patients who were $<6$ years old was similar to older patients (14 versus 10 survivors; HR:.483, 95\% CI: .157-1.488; $P=.205$ ). Cumulative mortality was higher in hypertensive (51.4\%) than nonhypertensive $(40.9 \%)$ CRS, but the difference did not reach statistical significance (HR:.476, 95\% CI: .183$1.240 ; P=.129)$. Survival comparison between non-CRS (controls) and CRS patients revealed no statistically significant difference (HR:.496, 95\% CI: .239-1.031; Figure 1). Figure 2 shows that patients with Type 5 CRS survived better than Type 3 CRS (HR:.479, 95\% CI: .299-.768). The cumulative survival for $\mathrm{MAH}$, septicaemia, and acute glomerulonephritis was $81.4 \%, 39.8 \%$, and $21.4 \%$, respectively; none of the patients with AKI due to hypovolaemia, frusemide, captopril, and leukaemia survived. CRS due to aetiologies other than AGN was significantly less associated with mortality compared with CRS due to AGN (40.4\% versus $78.6 \%$; HR: .544; 95\% CI: .322-.919; $P=.023$ ). A pairwise comparison statistics (Wilcoxon) revealed that MAH survived significantly better than other CRS causes $(P=.014)$. No death occurred in AKI-1, but there were 2 and 15 deaths in AKI-2 and AKI-3, respectively; AGN-AKI had one death. Mortality was similar in all AKI stages (HR: 1.872, 95\% CI: .761-4.603) as well as in all the three AKI types (HR: 1.385, 95\% CI: .7992.400) of CRS. Survival comparisons among the AKI types in both CRS and non-CRS revealed no significant differences 


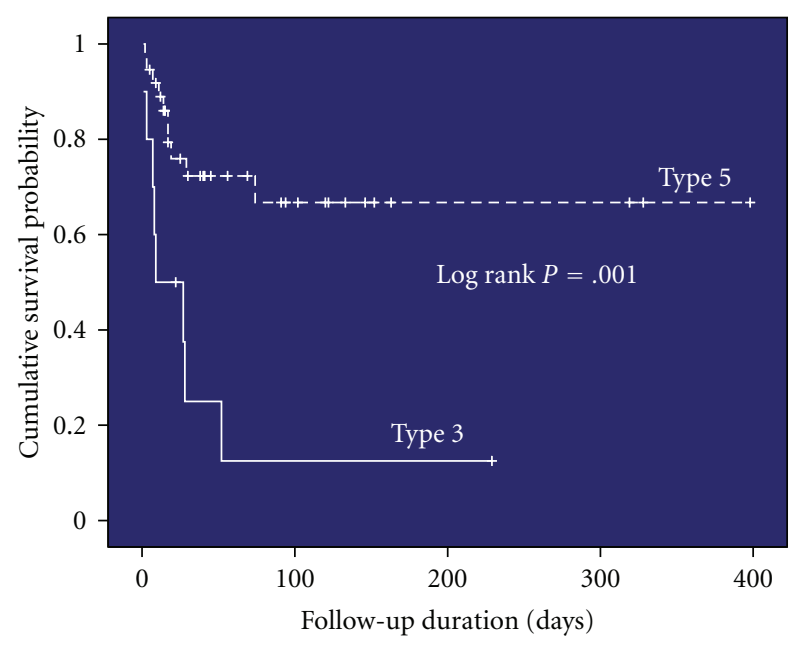

FIGURE 2: Kaplan-Meier survival curves showing significantly better survival in Type 5 compared to Type 3 cardiorenal syndrome (72.3\% versus $12.5 \%)$.

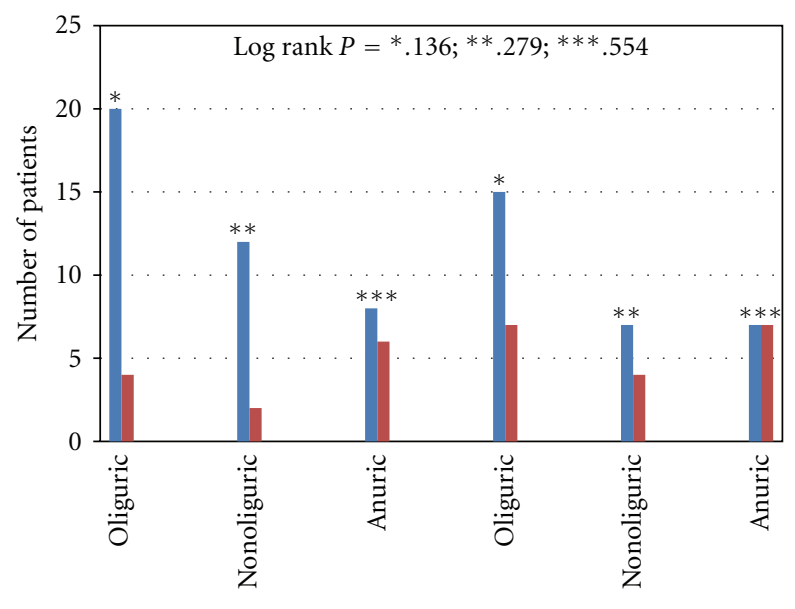

Controls Cardiorenal syndrome

- Survived

- Died

FIgURE 3: Comparisons between controls and patients with cardiorenal syndrome with regard to survival in the acute kidney injury (AKI) types. The number of patients surviving in oliguric, nonoliguric, and anuric AKI was similar in both groups of patients.

(Figure 3). Figure 4 shows that mortality in nonanaemic CRS patients was significantly higher compared to anaemic CRS (HR: 4.637, 95\% CI: 1.496-14.370). The outcome was significantly better among CRS patients who required no dialysis than those who required dialysis (HR: .284, 95\% CI:.106-.765; Figure 5). A stratification to CRS type showed that dialysis-requiring Type 3 CRS had significantly higher cumulative mortality rate than dialysis-requiring Type 5 CRS ( $100 \%$ versus $51.1 \%$; Log rank $P=.049$ ). This was similarly so for those not requiring dialysis (Type 3 CRS: $70.0 \%$

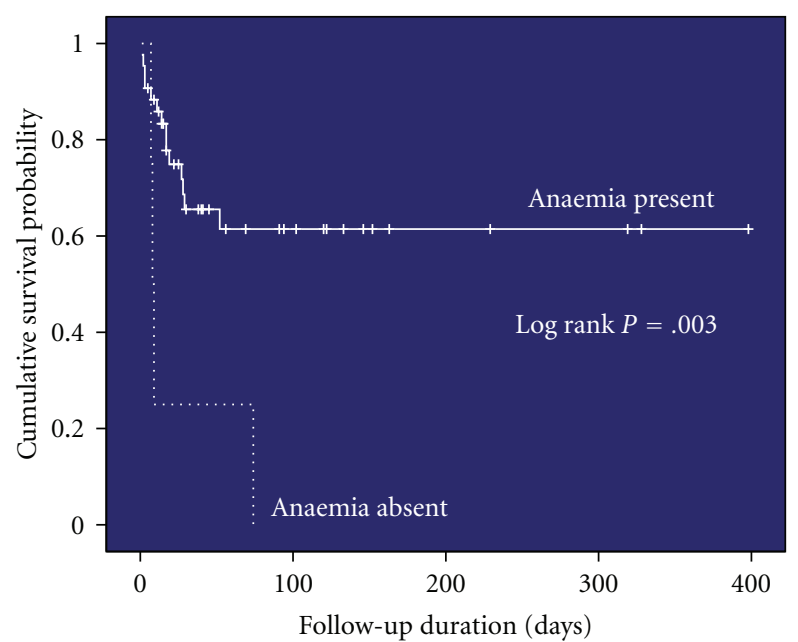

FIgURE 4: Kaplan-Meier survival curves comparing survival in cardiorenal syndrome patients with anaemia and those without anaemia. Patients with anaemia had significantly higher survival rate compared to nonanaemic patients (61.4\% versus $25.0 \%$ ).

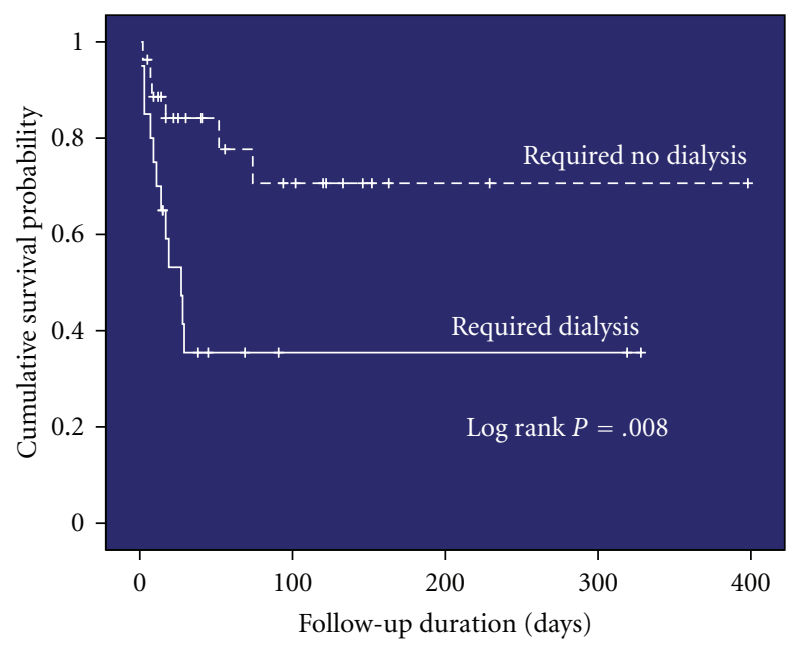

Figure 5: Kaplan-Meier survival curves for dialysis-requiring and non-dialysis requiring cardiorenal syndrome patients. Dialysisrequiring-cardiorenal syndrome had significantly lower survival rate compared to those who required no dialysis $(35.5 \%$ versus $70.6 \%)$.

versus Type 5 CRS: $19.1 \%$ mortality; $\log \operatorname{rank} P=.039)$. Further stratification for aetiology showed poorer survival with regards to Type 3 CRS compared to Type 5 as all Type 3 patients who required dialysis died $(n=5)$; all had AGN that was associated with poorer outcome compared to either MAH or septicaemia (Wilcoxon, $P=.001$ ). Eighteen CRS patients died at the end of 398 follow-up days, while 28 survived, and one took voluntary discharge. The cumulative CRS-specific mortality was $45.7 \%$. Thirty 
patients died overall (both CRS and controls) thus bringing the cumulative all-cause-mortality to $34.2 \%$.

\section{Discussion}

This study revealed CRS as a highly prevalent clinical event in acute perturbation of kidney function with hypertension as a common cardiovascular comorbidity. Given the spectrum of CRS aetiology in this study, hypertension was probably the result of intravascular congestion brought about by oliguria seen in $76.6 \%$ of the patients on one hand, and activation of the renin-angiotensin-aldosterone-system following renal hypoperfusion secondary to acute proliferative changes of AGN on the other hand. Interestingly, hypertension was found not to be a significant risk factor for mortality in this study (HR: 0.476, 95\% CI: 0.183-1.240).

The acute nature of the CRS and the accompanying hypertension, as well as, prompt response to antihypertensive treatment, and vascular decongestion following diuretic phase onset could be responsible for this. Left ventricular hypertrophy and congestive HF are common in childhood AGN and CKD with high mortality rate [7, 1416]. In this study, HF was highly prevalent and mortality rate from CRS was equally high but was not significantly different from controls; this is not withstanding the fact that the HF was of the severest class (Class IV). Anaemia is a highly prevalent comorbidity in both AKI $[5,6,17]$ and CKD [18]; it has been associated with increased severity of congestive HF, increased hospitalization, worse cardiac function and functional class, the need for higher doses of diuretics, progressive worsening of renal function, and reduced quality of life [19]. Anaemia occurred in $91.5 \%$ of our patients with anaemia-specific mortality rate of $38.6 \%$. Nonanaemic CRS patients were, however, 4.6 times more likely to die than their anaemic counterparts (95\% CI: 1.496-14.370). This is because the majority of the anaemic cases were due to malaria that responded rapidly to treatment; the acute nature of the accompanying congestive anaemic HF that responded promptly to blood transfusion also contributed to better survival in the anaemic CRS compared to nonanaemic CRS that was largely due to poor outcome septicaemia and AGN. While AKI stage and type did not influence the outcome in this study, Type 5 CRS was found to protect against mortality. The positive impact of Type 5 CRS on survival was due to the fact that the majority of the patients in this class had MAH that was significantly associated with the highest survival rate compared to Type 3 CRS in which majority of the patients had AGN that was significantly associated with very low survival rate. The fact that CRS patients who required no dialysis survived better than those who required dialysis (HR: 0.28 ; 95\% CI: $0.106-0.765$ ) could mean more severe structural kidney pathology and dysfunction in those needing dialysis. This was particularly more evident when CRS was stratified for CRS types and outcome.

It is concluded that CRS was a very common clinical event with high mortality rate in critically ill children. Compared to controls, CRS mortality rate was not significantly higher; risk factors for mortality in CRS were CRS Type 3, AGN-associated CRS, dialysis-requiring CRS, and heart failure not associated with anaemia. Preventive measures aimed at some of the preventable aetiologies of CRS might be critical to reducing its prevalence.

\section{Conflict of Interests}

The authors declare that there is no conflict of interests.

\section{References}

[1] C. Ronco, P. McCullough, S. D. Anker et al., "Cardio-renal syndromes: report from the consensus conference of the acute dialysis quality initiative," European Heart Journal, vol. 31, no. 6, pp. 703-711, 2010.

[2] S. M. Bagshaw, D. N. Cruz, N. Aspromonte et al., "Epidemiology of cardio-renal syndromes: workgroup statements from the 7th ADQI Consensus Conference," Nephrology Dialysis Transplantation, vol. 25, no. 5, pp. 1406-1416, 2010.

[3] M. M. Mitsnefes, "Cardiovascular complications of pediatric chronic kidney disease," Pediatric Nephrology, vol. 23, no. 1, pp. 27-39, 2008.

[4] A. M. Katz, Heart Failure Pathophysiology, Molecular Biology and Clinical Management, Lippincott Williams \& Wilkins, Philadelphia, Pa, USA, 2000.

[5] W. A. Olowu and K. A. Adelusola, "Pediatric acute renal failure in southwestern Nigeria," Kidney International, vol. 66, no. 4, pp. 1541-1548, 2004.

[6] W. A. Olowu, J. B. E. Elusiyan, S. A. Badejo, and O. A. Adenowo, "Acute renal failure in African children with Burkitt's lymphoma: a comparison of two treatment regimens," Pediatric Blood and Cancer, vol. 46, no. 4, pp. 446-453, 2006.

[7] I. Anochie and F. Eke, "Chronic renal failure in children: a report from Port Harcourt, Nigeria (1985-2000)," Pediatric Nephrology, vol. 18, no. 7, pp. 692-695, 2003.

[8] J. F. Price, A. R. Mott, H. A. Dickerson et al., "Worsening renal function in children hospitalized with decompensated heart failure: evidence for a pediatric cardiorenal syndrome?" Pediatric Critical Care Medicine, vol. 9, no. 3, pp. 279-284, 2008.

[9] R. L. Mehta, J. A. Kellum, S. V. Shah et al., "Acute Kidney Injury Network: report of an initiative to improve outcomes in acute kidney injury," Critical Care, vol. 11, no. 2, article R31, 2007.

[10] National Kidney Foundation, "K/DOQI clinical practice guidelines for chronic kidney disease: evaluation, classification and stratification," American Journal of Kidney Disease, vol. 39, supplement 1, pp. S76-S92, 2002.

[11] R. Bellomo, C. Ronco, J. A. Kellum, R. L. Mehta, and P. Palevsky, "Acute renal failure-definition, outcome measures, animal models, fluid therapy and information technology needs: the Second International Consensus Conference of the Acute Dialysis Quality Initiative (ADQI) Group," Critical Care, vol. 8, no. 4, pp. R204-212, 2004.

[12] D. T. Hsu and G. D. Pearson, "Heart failure in children part I: history, etiology, and pathophysiology," Circulation: Heart Failure, vol. 2, no. 1, pp. 63-70, 2009.

[13] National High Blood Pressure Education Programme Working Group on Hypertension Control in children and Adolescents, "Update on the 1987 task force report on high blood pressure in children and adolescents," Pediatrics, vol. 98, no. 4, pp. 649658, 1996. 
[14] W. A. Olowu, "Systemic complications of acute glomerulonephritis in Nigerian children," The Nigerian postgraduate medical journal, vol. 9, no. 2, pp. 83-87, 2002.

[15] R. S. Parekh, C. E. Carroll, R. A. Wolfe, and F. K. Port, "Cardiovascular mortality in children and young adults with end-stage kidney disease," Journal of Pediatrics, vol. 141, no. 2, pp. 191-197, 2002.

[16] J. W. Groothoff, M. P. Gruppen, M. Offringa et al., "Mortality and causes of death of end-stage renal disease in children: a dutch cohort study," Kidney International, vol. 61, no. 2, pp. 621-629, 2002.

[17] I. C. Anochie and F. U. Eke, "Acute renal failure in Nigerian children: port Harcourt experience," Pediatric Nephrology, vol. 20, no. 11, pp. 1610-1614, 2005.

[18] S. M. Koshy and D. F. Geary, "Anemia in children with chronic kidney disease," Pediatric Nephrology, vol. 23, no. 2, pp. 209219, 2008.

[19] D. S. Silverberg, D. Wexler, A. Iaina, S. Steinbruch, Y. Wollman, and D. Schwartz, "Anemia, chronic renal disease and congestive heart failure - the cardiorenal anemia syndrome: the need for cooperation between cardiologists and nephrologists," International Urology and Nephrology, vol. 38, no. 2, pp. 295310, 2006 


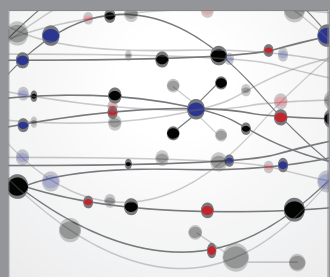

The Scientific World Journal
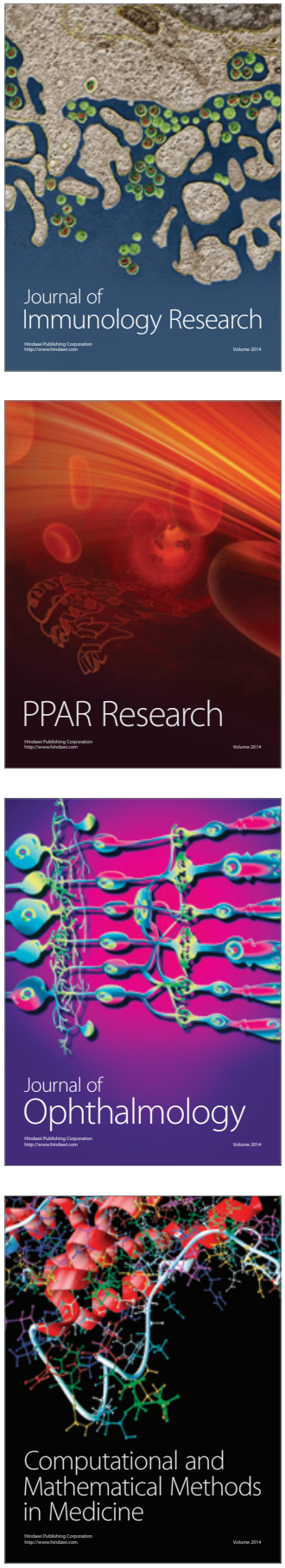

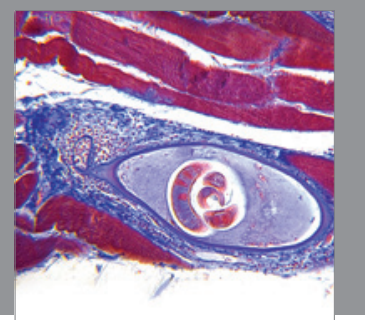

Gastroenterology

Research and Practice
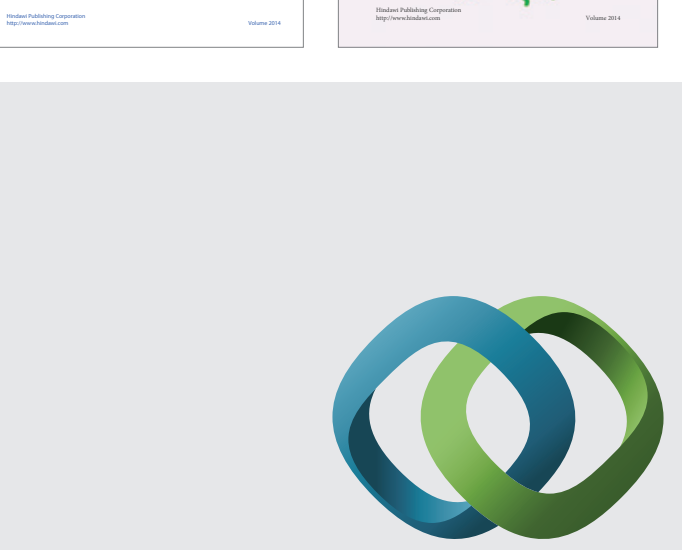

\section{Hindawi}

Submit your manuscripts at

http://www.hindawi.com
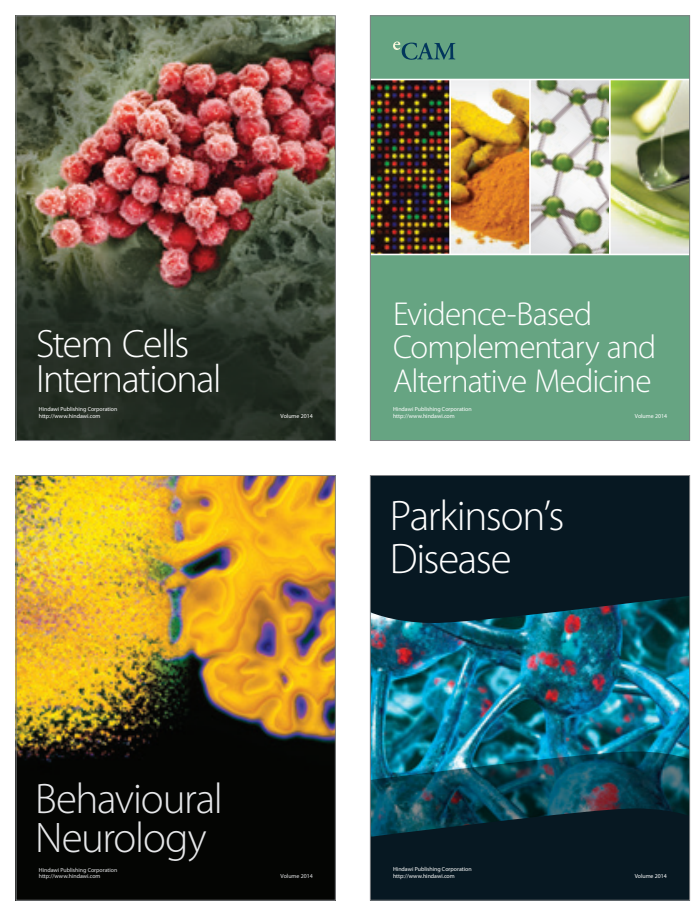

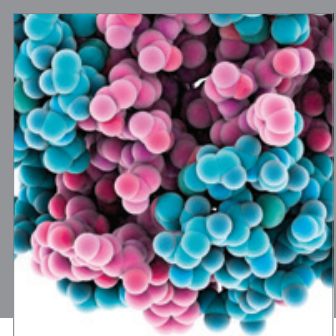

Journal of
Diabetes Research

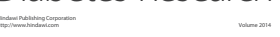

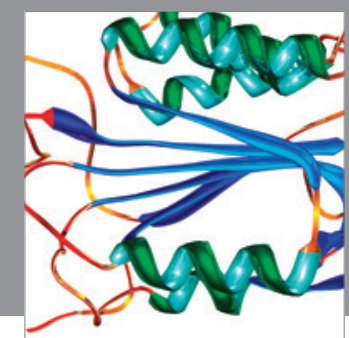

Disease Markers
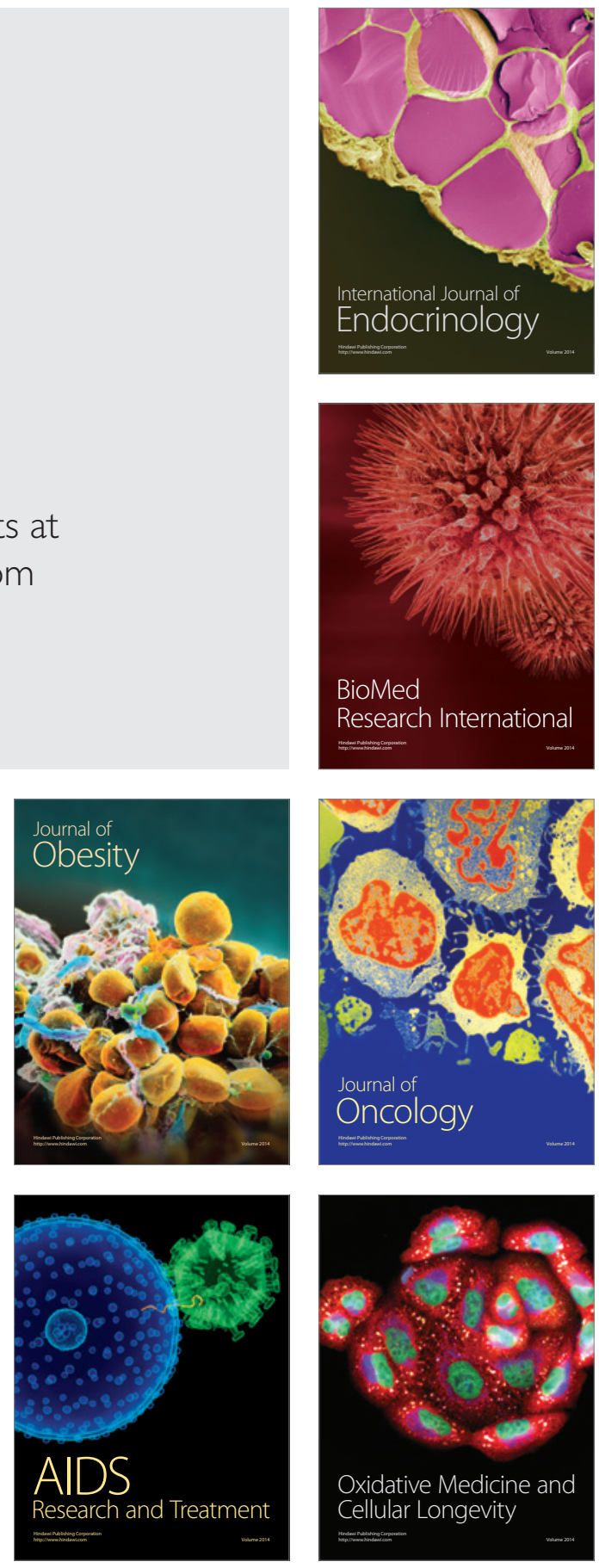